\title{
Molar Heat Capacity of AgI: Evaluation of the Cationic and Anionic Contributions from Thermoelectric Power Data
}

\author{
Alberto Schiraldi and Primo Baldini \\ Dipartimento di Chimica Fisica dell'Università di Pavia, Pavia (Italy) \\ Z. Naturforsch. 39a, 682-685 (1984); received April 16, 1984

\begin{abstract}
Thermoelectric power data provide a useful tool for the evaluation of the thermodynamic
This is the case of AgI, whose standard modifications have been investigated with silver

The interpretation of the thermoelectric power change at the melting point allows one to

The analysis of the thermoelectric power change at the transition point, from the wurtzitic to

The summation of the ionic contributions so computed reproduces rather satisfactorily the
\end{abstract} \\ properties of ionic species of both solid and molten electrolytes in equilibrium with electrodes of \\ thermocells; however a previous evaluation of the heat of transport of the mobile ions is \\ necessary. \\ electrode thermocells some years ago. \\ recognize that the heat of transport of the silver ions in the high conductivity bcc modification is \\ practically the same as in molten AgI. This result has been used to evaluate the molar heat of the \\ silver ions in the bcc phase from the corresponding thermoelectric power data reported in the \\ literature. \\ the bcc phase, as well as a more speculative interpretation of thermoelectric power data obtained \\ with iodine electrode thermocells, allows one to evaluate the molar heat of the iodide ions in the \\ bcc modification of AgI. \\ trend of AgI molar heat data obtained with adiabatic calorimetry.
}

\section{Introduction}

In 1929 Reinhold [1] did show that thermoelectric power determinations a simple salt, AX, may be related to the EMF of the corresponding galvanic cell of formation, viz.,

$$
\begin{aligned}
\varepsilon_{1} & =S(\mathrm{~A})-S\left(\mathrm{~A}^{+}\right)-Q / T \\
\varepsilon_{2} & =S\left(\mathrm{X}^{-}\right)-\frac{1}{2} S\left(\mathrm{X}_{2}\right)-Q / T, \\
\varepsilon_{2}-\varepsilon_{1} & =f \mathrm{dEMF} / \mathrm{d} T= \\
& =\left[S\left(\mathrm{~A}^{+}\right)+S\left(\mathrm{X}^{-}\right)\right]-\left[S(\mathrm{~A})+\frac{1}{2} S\left(\mathrm{X}_{2}\right)\right], \\
& \quad(f=\text { Faraday constant })
\end{aligned}
$$

where $\varepsilon$ is the thermoelectric power, the subscripts 1 and 2 refer to thermocells with electrodes reversible to $\mathrm{A}^{+}$and to $\mathrm{X}^{-}$, respectively, $S(\mathrm{~A})$ and $S\left(\mathrm{~A}^{+}\right)$are the molar entropies of the metal $\mathrm{A}$ of the electrode and of the $\mathrm{A}^{+}$ions of the electrolyte (analogous definition applies to $S\left(\mathrm{X}_{2}\right)$ and to $\left.S\left(\mathrm{X}^{-}\right)\right)$and $Q$ is the heat of transport of the mobile ionic species (generally the cation). Equations (1) and (2) hold only when $\mathrm{AX}$ is a pure ionic conductor, no matter if it is solid or molten.

Reprint requests to Herrn Dr. Alberto Schiraldi, Dipartimento di Chimica Fisica, Università di Pavia, Viale Taramelli 16, 27100 Pavia (Italy).
In the present work a simple extension of Reinhold's relationship (3) is proposed, viz:

$$
\begin{aligned}
T\left[\mathrm{~d}\left(\varepsilon_{2}-\varepsilon_{1}\right) / \mathrm{d} T\right]= & {\left[C_{p}\left(\mathrm{~A}^{+}\right)+C_{p}\left(\mathrm{X}^{-}\right)\right] } \\
& -\left[C_{p}(\mathrm{~A})+\frac{1}{2} C_{p}\left(\mathrm{X}_{2}\right)\right]
\end{aligned}
$$

which allows one to evaluate the molar heat capacity at constant pressure of $\mathrm{AX}$,

$$
C_{p}(\mathrm{AX})=\left[C_{p}\left(\mathrm{~A}^{+}+C_{p}\left(\mathrm{X}^{-}\right)\right],\right.
$$

when the $C_{p}$ 's of the parent elements are known.

However, more significant would be an approach to the single ionic contributions, viz. $C_{p}\left(\mathrm{~A}^{+}\right)$and $C_{p}\left(\mathrm{X}^{-}\right)$, through a previous evaluation of the heat of transport $Q$.

It must be mentioned that the relation by WagnerPitzer (WP in the following) $[2,3]$ for the single ion contributions to the overall molar entropy of a salt would provide a more direct approach to $C_{p}\left(\mathrm{~A}^{+}\right)$ and $C_{p}\left(\mathrm{X}^{-}\right)$in the case of a simple ionic melt and of an ionic solid with Frenkel point defects. The WP, however, would not be valid for the so called "solid electrolytes", such as $\alpha-\mathrm{AgI}, \mathrm{RbAg}_{4} \mathrm{I}_{5}$ and similar compounds, as well as for the high ionic conductivity glasses, such as those obtained from AgI and Ag oxysalt [4], mainly because of their structural disorder, Accordingly, the procedure via

0340-4811/84/0700-0682 \$ 01.30/0. - Please order a reprint rather than making your own copy. 
thermoelectric power data might be of some relevance for these materials.

Thermoelectric power data for all the three standard modifications of AgI, obtained either with Ag or with $\mathrm{I}_{2}$ electrode thermocells, are reported in the literature [5-9].

These have been employed in the present work to compute $C_{p}\left(\mathrm{Ag}^{+}\right)$and $C_{p}\left(\mathrm{I}^{-}\right)$. The corresponding $C_{p}(\mathrm{AgI})=\left[C_{p}\left(\mathrm{Ag}^{+}\right)+C_{p}\left(\mathrm{I}^{-}\right)\right]$values for the $\alpha$ phase are compared with the results of adiabatic calorimetry found in the literature. Analogous quantities for the other phases of $\mathrm{AgI}$ have been obtained via WP. These results allow one to obtain also the corresponding trends of the molar ionic enthalpy vs. $T$.

\section{Evaluation of the heat of transport}

In previous papers, cf. [10], it has been suggested that the classic [11] relationship

$$
Q \geqq E_{\text {act }} \gg k T
$$

for the migration of a single kind of point defect, where $E_{\text {act }}$ is the conductivity or selfdiffusion activation energy, may be reasonably replaced by the "numerical" equality

$$
Q \sim E_{\text {act }}
$$

for many solid electrolytes. However, in some cases, e.g. $\alpha$-AgI, the experimental $E_{\text {act }}$ is so close to $k T$ that it cannot be assumed to represent any simple physical process, e.g. the jump of a single $\mathrm{Ag}^{+}$ion to the next neighbouring lattice site. Accordingly, the expression (5), which is still based upon the "hopping mechanism" (simply amended for terms concerning the formation of point defects), should not be directly extended to solid electrolytes with $E_{\text {act }} \sim k T$.

For these compounds a tentative evaluation of the phenomenological quantity $Q$ should be approached through different assumptions, suggested by some experimental evidence, e.g. by the variation of the thermoelectric power at phase transitions, or by the expected order of magnitude of the single ion contribution to the overall molar heat capacity.

\section{The case of AgI}

The experimental thermoelectric power data reported in the literature $[6,8,9]$ are employed in the present work and fitted as

$$
\begin{aligned}
& \varepsilon_{1 \beta}=\left(25.47-15.75 \cdot 10^{3} T^{-1}\right) R, \quad[6], \\
& \varepsilon_{1 \alpha}=\left(-4.29-1.45 \cdot 10^{3} T^{-1}\right) R, \quad[6], \\
& \varepsilon_{1 I}=\left(-6.02+8.71 \cdot 10^{-4} T\right) R, \quad[8], \\
& \varepsilon_{2 \chi}=\left(-11.46-2.91 \cdot 10^{3} T^{-1}\right) R, \quad[9],
\end{aligned}
$$

where 1 and 2 refer to thermocells with $\mathrm{Ag}$ or $\mathrm{I}_{2}$ electrodes, respectively, $\beta, \alpha$ and $I$ indicate the $\mathrm{AgI}$ modification, $R$ is the gas constant and $T$ is dimensionless in numerical combinations such as these.

The evaluation of the heat of transport, $Q_{\alpha}$, in the expressions (1) and (2) for $\varepsilon_{1 \alpha}$ and $\varepsilon_{2 \alpha}$ has been here achieved by working out the difference $\left(\varepsilon_{1 I}-\varepsilon_{1 \alpha}\right)$ at the melting point, $T_{\text {fus }}=830 \mathrm{~K}$; according to the literature $[7,8]$, this difference is indeed negligible, so that one has

$$
\begin{aligned}
\left(\varepsilon_{1 I}-\varepsilon_{1 \alpha}\right)= & {\left[S\left(\mathrm{Ag}^{+}, \alpha\right)-S\left(\mathrm{Ag}^{+}, I\right)\right]_{T_{\text {fus }}} } \\
& +\left(Q_{\alpha}-Q_{I}\right) / T_{\text {fus }} \sim 0 .
\end{aligned}
$$

This experimental result is here justified by assuming that no contribution is due to the "fusion" of the $\mathrm{Ag}^{+}$sublattice, so that

$$
\left[S\left(\mathrm{Ag}^{+}, \alpha\right)-S\left(\mathrm{Ag}^{+}, I\right)\right]_{T_{\text {fus }}} \sim 0
$$

and

$$
Q_{\chi} \sim Q_{I}=14.6 \mathrm{~kJ} \mathrm{~mol}^{-1},
$$

where the $Q_{I}$ value has been obtained [8] through WP. With this $Q_{\alpha}$ value one has

$$
\begin{aligned}
C_{p}\left(\mathrm{Ag}^{+}, \alpha\right) & =C_{p}(\mathrm{Ag})-T d \varepsilon_{1 \alpha} / \mathrm{d} T+Q_{\alpha} / T \\
& =\left(\frac{18100}{T^{2}}+\frac{312}{T}+2.57+0.001 T\right) R,
\end{aligned}
$$

where (6) has been directly drawn from (1) by assuming $Q_{\alpha}$ as $T$ independent.

Both $C_{p}\left(\mathrm{Ag}^{+}, \beta\right)$ and $C_{p}\left(\mathrm{Ag}^{+}, I\right)$ may be obtained via $\mathrm{WP}$, viz.

$$
\begin{aligned}
& C_{p}\left(\mathrm{Ag}^{+}, \beta\right)=\left(5.51-0.015 T+2.77 \cdot 10^{-5} T^{2}\right) R, \\
& C_{p}\left(\mathrm{Ag}^{+}, I\right)=3.84 R,
\end{aligned}
$$

from the values of the AgI molar entropy reported in [12] and [13], respectively.

Thermoelectric power data, however, allow one to extend the knowledge of the thermodynamic properties of the $\mathrm{Ag}^{+}$sublattice through an analysis of 
the difference $\left(\varepsilon_{1 \beta}-\varepsilon_{1 \alpha}\right)_{T_{x \beta}}$,

$$
\begin{aligned}
\left(\varepsilon_{1 \beta}-\varepsilon_{1 \alpha}\right)_{T_{\alpha, \beta}}= & {\left[S\left(\mathrm{Ag}^{+}, \alpha\right)-S\left(\mathrm{Ag}^{+}, \beta\right)\right]_{T_{x, \beta}} } \\
& -\left(Q_{\beta}-Q_{\alpha}\right) / T_{\alpha, \beta}=-4.3 R .
\end{aligned}
$$

Because of the variation of the transport numbers of the Frenkel defects in $\beta$-AgI, $Q_{\beta}$ does depend on $T$, viz.

$$
Q_{\beta}=t_{\mathrm{i}}\left(E_{\mathrm{f}} / 2+U_{\mathrm{i}}\right)-t_{\mathrm{v}}\left(E_{\mathrm{f}} / 2+U_{\mathrm{v}}\right),[2],
$$

where $E_{\mathrm{f}}$ is the formation energy for a Frenkel pair and $U_{\mathrm{i}}, U_{\mathrm{v}}, t_{\mathrm{i}}$ and $t_{\mathrm{v}}$ are the migration energies and the transport numbers of interstitial ions (subscript "i") and cationic vacancies (subscript "v"), respectively. In a previous paper [6], it was found that

$$
Q_{\beta}=\left(2 t_{\mathrm{i}}-1\right) 82.4 \mathrm{~kJ} \mathrm{~mol}^{-1},
$$

where the $t_{1}$ values reported there have been fitted here as

$$
t_{\mathrm{i}}=1.31-1.41 \cdot 10^{-3} T,
$$

so that

$$
Q_{\beta}=(134-0.233) \mathrm{kJ} \mathrm{mol}^{-1} .
$$

This value, introduced into (9), give

$$
\begin{aligned}
& T_{\chi, \beta}\left[S\left(\mathrm{Ag}^{+}, \alpha\right)-S\left(\mathrm{Ag}^{+}, \beta\right)\right]_{T_{x, \beta}} \\
& =\Delta H_{\alpha, \beta}\left(\mathrm{Ag}^{+}\right)=6.14 \mathrm{~kJ} \mathrm{~mol}^{-1}
\end{aligned}
$$

which, due to the result of [12] (viz. $\Delta H_{\alpha, \beta}(\mathrm{AgI})$ $=6.14 \pm 0.3 \mathrm{~kJ} \mathrm{~mol}^{-1}$ ) allows one to conclude that the transition enthalpy change concerns only the $\mathrm{Ag}^{+}$ sublattice.

According to the above statements it follows that

$$
\left[S\left(\mathrm{Ag}^{+}, \alpha\right)\right]_{T_{\text {fus }}} \sim\left[S\left(\mathrm{Ag}^{+}, I\right)\right]_{T_{\text {fus }}},
$$

i.e.

$$
\left[H\left(\mathrm{Ag}^{+}, \chi\right)\right]_{T_{\text {fus }}} \sim\left[H\left(\mathrm{Ag}^{+}, I\right)\right]_{T_{\text {fus }}},
$$

while, from (6) and (8) one has

$$
C_{p}\left(\mathrm{Ag}^{+}, \alpha\right) \sim C_{p}\left(\mathrm{Ag}^{+}, I\right) \text { for } T>T_{\alpha, \beta} .
$$

Thence, the process occurring at $T_{\alpha . \beta}$ may be considered as the "fusion" of the $\mathrm{Ag}^{+}$sublattice within the network of the $\mathrm{I}^{-}$anions, which simultaneously undergoes a second order transition (see below). The latter sublattice melts at $T=T_{\text {fus }}$ with an enthalpy change corresponding to the whole $\Delta H_{\text {fus }}$ (AgI) (viz. $\left.9.4 \mathrm{~kJ} \mathrm{~mol}^{-1}[12]\right)$.

In the liquid state, according to $\mathrm{PW}$

$$
S\left(\mathrm{Ag}^{+}, I\right) \sim S\left(\mathrm{I}^{-}, I\right),
$$

i.e.

$$
C_{p}\left(\mathrm{Ag}^{+}, I\right) \sim C_{p}\left(\mathrm{I}^{-}, I\right)
$$

i.e.

$\left[H\left(\mathrm{Ag}^{+}, I\right)\right]_{T_{\text {fus }}}=\left[H\left(\mathrm{I}^{-}, I\right)\right]_{T_{\text {fus }}}+C_{\alpha}$

$$
=\left[H\left(\mathrm{Ag}^{+}, \alpha\right)\right]_{T_{\text {fus }}}=\left[H\left(\mathrm{I}^{-}, \alpha\right)\right]_{T_{\text {fus }}}+\Delta H_{\text {fus }}+C_{\alpha},
$$

where $C_{\gamma}$ is an integration constant. Thus,

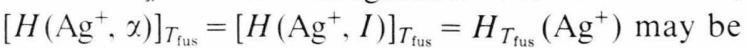
chosen as a suitable reference value for the enthalpy of either sublattice, viz.

$H\left(\mathrm{Ag}^{+}, \alpha\right)=H_{T_{\text {fus }}}\left(\mathrm{Ag}^{+}\right)-\int_{T>T_{\text {x. }}}^{T_{\text {fus }}} C_{p}\left(\mathrm{Ag}^{+}, \alpha\right) \mathrm{d} T$ and

$H\left(\mathrm{I}^{-}, \chi\right)=H_{T_{\text {fus }}}\left(\mathrm{Ag}^{+}\right)-\Delta H_{\text {fus }}-C_{\chi}$

$$
-\int_{T>T_{\text {x. }}}^{T_{\text {fus }}} C_{p}\left(\mathrm{I}^{-}, \boldsymbol{\alpha}\right) \mathrm{d} T,
$$

which gives

$$
\begin{aligned}
& {\left[H\left(\mathrm{Ag}^{+}, \alpha\right)-H\left(\mathrm{I}^{-}, \chi\right)\right]_{T_{\alpha, \beta}}=\Delta H_{\alpha, \beta}+C_{\beta}} \\
& \quad=\Delta H_{\mathrm{fus}}+C_{\gamma}-\int_{T_{x, \beta}}^{T_{\text {fus }}}\left[C_{p}\left(\mathrm{Ag}^{+}, \chi\right)-C_{p}\left(\mathrm{I}^{-}, \chi\right)\right] \mathrm{d} T,
\end{aligned}
$$

where $C_{\beta}$ is a constant of integration analogous to $C_{x}$.

Finally

$$
\begin{aligned}
& \int_{T_{x, \beta}}^{T_{\text {fus }}}\left[C_{p}\left(\mathrm{Ag}^{+}, \alpha\right)-C_{p}\left(\mathrm{I}^{-}, \alpha\right)\right] \mathrm{d} T \\
& =\left(\Delta H_{\mathrm{fus}}-\Delta H_{\gamma_{, \beta}}\right)+\left(C_{\alpha}-C_{\beta}\right) \sim 3.14 \mathrm{~kJ} \mathrm{~mol}^{-1},
\end{aligned}
$$

if one neglects the difference $\left(C_{\alpha}-C_{\beta}\right)$ and uses $\Delta H_{\text {fus }}$ and $\Delta H_{\alpha, \beta}$ of [12].

Thus $(6-8),(10)$ and (11) allow one to obtain the $H\left(\mathrm{Ag}^{+}\right)$and $H\left(\mathrm{I}^{-}\right)$trends vs. $T$ for all the three standard modifications of AgI [see Figure 1].

As $C_{p}\left(\mathrm{Ag}^{+}, \alpha\right) \sim 3.85 R$ between $T_{\alpha, \beta}$ and $T_{\text {fus }}$, from (11) one estimates

$$
\int_{T_{x, j}}^{F_{\text {fus }}} C_{p}\left(\mathrm{I}^{-}, x\right) \mathrm{d} T \sim 9.95 \mathrm{~kJ} \mathrm{~mol}^{-1},
$$

which gives

$$
C_{p}\left(\mathrm{I}^{-}, x\right) \sim 2.92 R=\mathrm{const},
$$

and, with (6),

$$
C_{p}(\alpha-\mathrm{AgI})=C_{p}\left(\mathrm{Ag}^{+}, \alpha\right)+C_{p}\left(\mathrm{I}^{-}, \alpha\right)=6.76 R,
$$

in agreement with the calorimetric findings [12, 14]. 
Since, according to (7) and to WP, $C_{p}\left(\mathrm{I}^{-}, \beta\right)_{T_{x, \beta}}$ $\sim C_{p}\left(\mathrm{Ag}^{+}, \beta\right)_{T_{x, \beta}}=3.97 R$, (12) would support the interpretation of the process occurring in the $\mathrm{I}^{-}$ sublattice as a second order transition (viz. $\Delta H=0$ and $\left.\Delta C_{p} \neq 0\right)$ with $\Delta C_{p}\left(\mathrm{I}^{-}\right) \sim R$. The direct procedure to attain $C_{p}\left(\mathrm{I}^{-}, \alpha\right)$ via the expression

$$
C_{p}\left(\mathrm{I}^{-}, \alpha\right)=\frac{1}{2} C_{p}\left(\mathrm{I}_{2}\right)+T\left[\mathrm{~d} \varepsilon_{2} \alpha / \mathrm{d} T\right]-Q_{\alpha} / T,
$$

analogous to (6), is complicated by the difficulty of a proper evaluation of $C_{p}\left(I_{2}\right)$. In the present case, the latter quantity does not correspond merely to the molar heat of gaseous $\mathrm{I}_{2}, C_{p}\left(\mathrm{I}_{2}, g\right)$, but to that of $\mathrm{I}_{2}$ adsorbed onto a Pt surface. A crude application of Langmuir's model would give

$$
C_{p}\left(\mathrm{I}_{2}\right)=C_{p}\left(\mathrm{I}_{2}, g\right)-L_{\mathrm{a}} / T,
$$

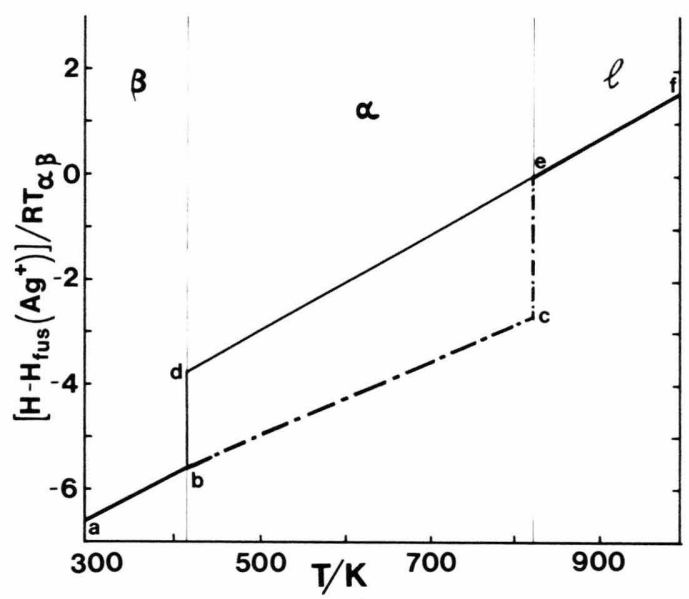

Fig. 1. Enthalpy of the $\mathrm{Ag}^{+}$and the $\mathrm{I}^{-}$sublattices in the three standard modifications of AgI, computed according to the equations of section 3. The enthalpy of the $\mathrm{Ag}^{+}$ sublattice at the melting point (point e) has been chosen as zero. The straight lines $\mathrm{a}, \mathrm{b}$ and $\mathrm{e}, \mathrm{f}$ correspond to the enthalpy of both $\mathrm{Ag}^{+}$and $\mathrm{I}^{-}$in the beta and in the liquid phase, respectively; the straight lines $b, d$, e and $b$, c, e correspond to the enthalpy trend at the phase transition and throughout the alpha phase for $\mathrm{Ag}^{+}$and $\mathrm{I}^{-}$, respectively.

[1] H. Reinhold, Z. anorg. allg. Chem. 171, 181 (1929). H. Reinhold and A. Blachny, Z. Elektrochem. 39, 290 (1933).

[2] K. S. Pitzer, J. Phys. Chem. 65, 147 (1961).

[3] C. Wagner, Progr. Sol. Stat. Chem. 7, 1 (1972).

[4] A. Schiraldi, Electrochim. Acta 23, 1039 (1978).

[5] K. Shahi and S. Chandra, Z. Naturforsch. 30 a, 1055 (1975).

[6] A. Schiraldi, Z. phys. Chem. N.F. 97, 285 (1975).

[7] A. Kvist, ibid. 19 a, 1159 (1964).

[8] A. Schiraldi and E. Pezzati, ibidem 33 a, 42 (1978).

[9] K. Wagener, Z. phys. Chem. N.F. 21, 74 (1959).

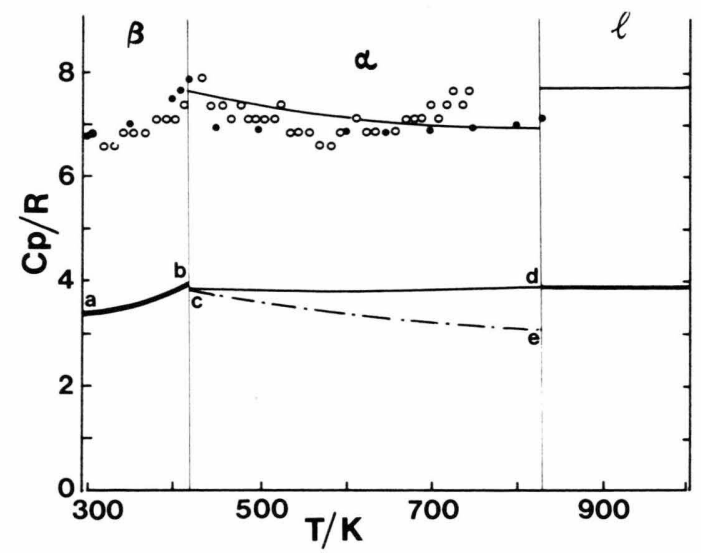

Fig. 2. Molar heat capacity of the three standard modifications of AgI: the curve drawn through the experimental data reported in the literature $[14,16]$ for the alpha phase is the sum of the c, d and c, e trends, evaluated in the present work and corresponding to the single sublattice contributions. The curve $\mathrm{a}, \mathrm{b}$ corresponds to both $\mathrm{Ag}^{+}$and $\mathrm{I}^{-}$molar heats in the beta phase: they were evaluated according to WP. Filled circles: [14]; open circles: [16]. The horizontal trend drawn for the liquid phase corresponds to the data of ref. [15] and gives the underlying curve with use of the Wagner-Pitzer relationship.

where $L_{\mathrm{a}}$ is the corresponding heat of adsorption (here assumed as $T$ independent). A further complication is due to the uncertainty of evaluating $T\left[\mathrm{~d} \varepsilon_{2 \alpha} / \mathrm{d} T\right]$ from the experimental $\varepsilon_{2 \alpha}$ data, obtained at various $I_{2}$ partial pressures [9]. The latter, when fitted vs. $T$, give indeed rather randomly scattered slopes. Nevertheless, with $C_{p}\left(\mathrm{I}_{2}, g\right)=4.5 R$ [15] and assuming $L_{\mathrm{a}} \sim 14.5 \mathrm{~kJ} \mathrm{~mol}^{-1}$ [16], one has

$$
C_{p}\left(\mathrm{I}^{-}, \alpha\right)=(2.26+655 / T) R
$$

with the mean $\mathrm{d} \varepsilon_{2 \alpha} / \mathrm{d} T$ value (viz. $\frac{27400}{T^{2}} \mathrm{~J} \mathrm{~mol}^{-1}$ $\left.\mathrm{K}^{-2},[9]\right)$.

The corresponding $C_{p}(\alpha-\mathrm{AgI})$ is in satisfactory agreement with the calorimetric trend (see Figure 2).

[10] A. Schiraldi, P. Baldini and E. Pezzati, Sol. Stat. Ionics 9/10, 1187 (1983).

[11] A. R. Allnatt and S. A. Rice, J. Chem. Phys. 33, 573 (1960); A. R. Allnatt, Z. Naturforsch. 26 a, 10 (1971).

[12] J. Nolting and D. Rein, Z. phys. Chem. N.F. 66, 150 (1969).

[13] GMELINS HANDBUCH, 8. Auflage, Teil B2, 249 (1972).

[14] S. Hoshino, J. Phys. Soc. Japan 12, 315 (1957).

[15] JANAF Tables (1961).

[16] A. W. Adamson, Physical Chemistry of Surfaces, Interscience Publ. 1967, 569. 